\title{
Prediction of localized aerosol deposition in a realistic replica of human airways using experimental data and numerical simulation
}

\author{
Frantisek Lizal ${ }^{1, *}$, Jakub Elcner ${ }^{1}$, Miloslav Belka ${ }^{1}$,Jan Jedelsky ${ }^{1}$, and Miroslav Jicha ${ }^{1}$ \\ ${ }^{1}$ Brno University of Technology, Faculty of Mechanical Engineering, Energy Institute, Technicka 2896/2, Brno 616 69, Czech Republic
}

\begin{abstract}
The presence of aerosol deposition hot-spots in human airways presumably contributes to development of various diseases. The overall aerosol deposition in human lungs can be predicted with sufficient accuracy nowadays. However, the prediction of localized aerosol deposition poses arduous challenge, namely in diseased lungs. Numerical simulation is considered to be a promising tool for the successful prediction. Yet, the validation of such simulations is difficult to perform, as not enough experimental data acquired using realistic airway replicas is available. This paper presents a first comparison of localized deposition measurement and simulation performed on the identical realistic geometry. The analysis indicates that both approaches yield similar results for low Reynolds number flows.
\end{abstract}

\section{Introduction}

Although the inhalation therapy is widely used among patients with asthma, cystic fibrosis or chronic obstructive pulmonary disease nowadays, its potential is far from being completely utilized. It could be used for delivery of pharmaceutical aerosols suitable for treatment of systemic diseases such as diabetes, or for targeted cancer treatment. However, the problem of current inhalation devices is that they cannot be easily tailored for each individual patient. Yet, especially human upper airways vary widely among people, which leads to strong variability in the delivered dose in the case that the same type of inhaler is used by all patients.

Moreover, despite the fact that the overall aerosol deposition in human lungs can be predicted with sufficient accuracy, the prediction of localized aerosol deposition poses arduous challenge, namely in diseased lungs. It should be emphasized, that the localized deposition prediction is essential not only for pharmaceutical aerosols, but, as several authors demonstrated [1,2], the presence of aerosol deposition hot-spots in human airways presumably contributes to development of various diseases, such as bronchial carcinoma.

It is obvious that the future of inhalation therapy lies in our ability to predict the localized deposition of particles in patient-specific lung geometries. One approach is to use stochastic lung deposition models. This type of simulation has been applied recently by Farkas et al. [3] to calculate the deposition distribution of two marketed aerosol drugs. Another approach utilizes the fast development of computational fluid and particle dynamics (CFPD). It seems achievable to calculate the precise local deposition in resolution of few $\mathrm{mm}^{2}$ in the near future. The advances in CFPD are tightly linked with the innovation of medical imaging techniques, as the acquisition of high-quality geometry of airways and the precise knowledge of boundary conditions remain challenging tasks even nowadays.

Many studies documenting the development of numerical simulations of flow and particle deposition in human lungs have been published in last two decades (see e.g. the reviews of Hofmann [2] or Rostami [4]). However, only rarely have they been performed on realistic lung geometries and validated by reliable experimental data on the identical geometries. Yet, the validation represents extremely important step and only validated simulations can be used for realistic predictions in pharmaceutical or toxicological applications.

The difficulty of acquiring the experimental data grounds in complicated and hardly accessible geometry of lungs. The most realistic way of validation would be the in vivo measurement of the patient whose lung geometry was used for the simulation. However, several problems complicate this option. First, the medical imaging methods often require the application of radioactive substances, while the higher dose of radiation applied the better resolution of the data. Regrettably, the radiation dose is limited due to its negative health effect. The methods that do not use radioactivity are usually even less precise in measurement of deposited particles location. Second, it is extremely difficult to obtain the high quality images, as the human body moves during the scanning and also the lung geometry and the location of deposited particles inevitably change in time due to the physiological processes in the body. Third, the whole process is also complicated by the administrative requirements (informed consent, ethical committee approval, etc.) which are necessary to prevent the public health.

\footnotetext{
* Corresponding author: lizal@,fme.vutbr.cz
} 
The above mentioned obstacles lead to the application of in vitro approach. It means that the real human lung geometry is used for fabrication of physical cast, that is used for experiments. After that, the simulations and experiments can be performed on the identical geometry. This method was used by our team and will be described later in detail.

Among the recent authors who validated numerical simulations by in vitro data we can mention Kerekes et al. [5], who focused on the flow field in a simplified model of tracheobronchial airways and validated their results by laser Doppler velocimetry (LDV). However, their geometry was simplified to facilitate the application of the LDV technique. Nonetheless, the curvature and tortuosity of airways is important to be able to simulate the flow division in the tracheobronchial tree correctly, as was documented e.g. by Bauer and Brucker [6]. Validated simulation of lung flow performed on the curved realistic geometry was published e.g. by Elcner et al. [7].

There are very few papers dealing with the CFPD simulation of localized particle deposition so far. We should mention the work of Holbrook et al. [8], who focused on the quality of the wall surface and its influence on the results.

In this paper we compare the first results of our numerical simulation and measured deposition of particles in a realistic model of human airways consisting of the oral cavity and following airways down to the seventh generation of branching.

\section{Methods}

The method for measurement of localized aerosol deposition presented in this paper divides the airways into segments of sizes between 2 to $5 \mathrm{~cm}$. It is generally possible to go down to the resolution of approximately $5 \times 5 \times 5 \mathrm{~mm}$, which corresponds to the maximal resolution of the positron emission tomography (PET) scanner. The numerical simulation is able to go even to the $\mu \mathrm{m}$-scale and indeed such a good resolution is needed for real understanding of the pathological processes connected with the deposition hot-spots in human lungs. However, even the cm-scale is a good step towards the validated maximal resolution of CFPD calculations.

\subsection{Experimental setup}

The experimental setup has been described in detail in [9], therefore only a brief description will be given here. The measurement was performed on a realistic replica of human airways, whose dimensions and development have been published in [10]. The geometry of airways used for fabrication of our model comes from a computed tomography $(\mathrm{CT})$ of the tracheobronchial tree excised from a cadaver (for detail please see Schmidt et al. [11]), which was combined with a 3D scan of the wax cast of the so called Lovelace Respiratory Research Institute (LRRI) "A" model [12]. The combined airway geometry was then processed in a computer aided design (CAD) software Rhinoceros to form a segmented physical model of human lungs (Fig. 1). The model was originally divided into segments to facilitate other experimental methods, such as optical counting of deposited particles. The deposited particles were consequently evaluated for each segment also in the case of the presented PET method, although this method could generally go down to smaller portions of the model.

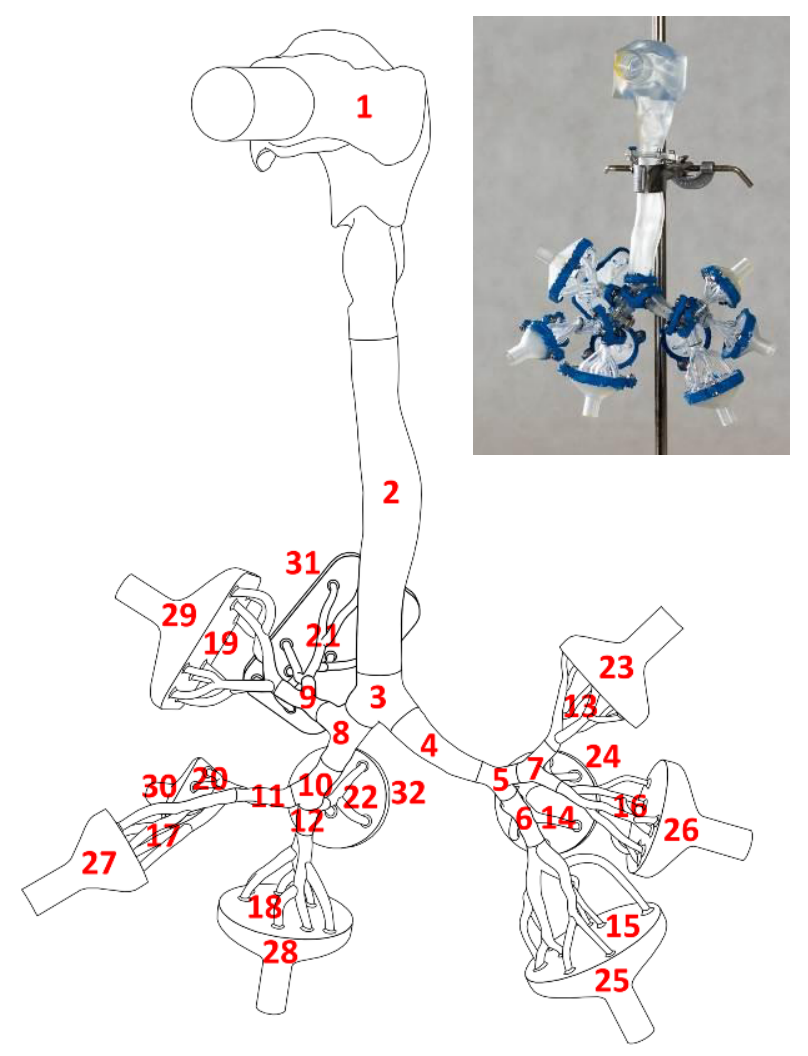

Fig. 1. A scheme with the designation of the segments and the photograph of a segmented realistic model of human lungs.

Both the experiment and the simulation were performed for steady inhalation flow rates of 15 and 30 liter/min. The whole experimental setup is schematically depicted in Fig. 2.

The realistic physical model was fixed in a wooden box and all ten outputs were connected to filters which were collecting the non-depositing particles. The aerosol particles in sizes of 2.5 and $4.3 \mu \mathrm{m}$ were generated using condensation monodisperse aerosol generator (CMAG) TSI 3475 and tagged by positron emitting isotope ${ }^{18} \mathrm{~F}$.

The exposed model was promptly transported to the hybrid PET/CT scanner Siemens Biograph Truepoint 64 and immediately scanned. Both the PET and CT images were used for evaluation in the software Carimas (Turku PET centre, Finland). Each segment of the model was enclosed in a separate volume of interest (VOI) in the Carimas software and its volume radioactivity in $\mathrm{Bq} / \mathrm{ml}$ was calculated. Consequently, the deposition fraction as a ratio of radioactivity in a given segment to the total radioactivity entering the model was determined. 


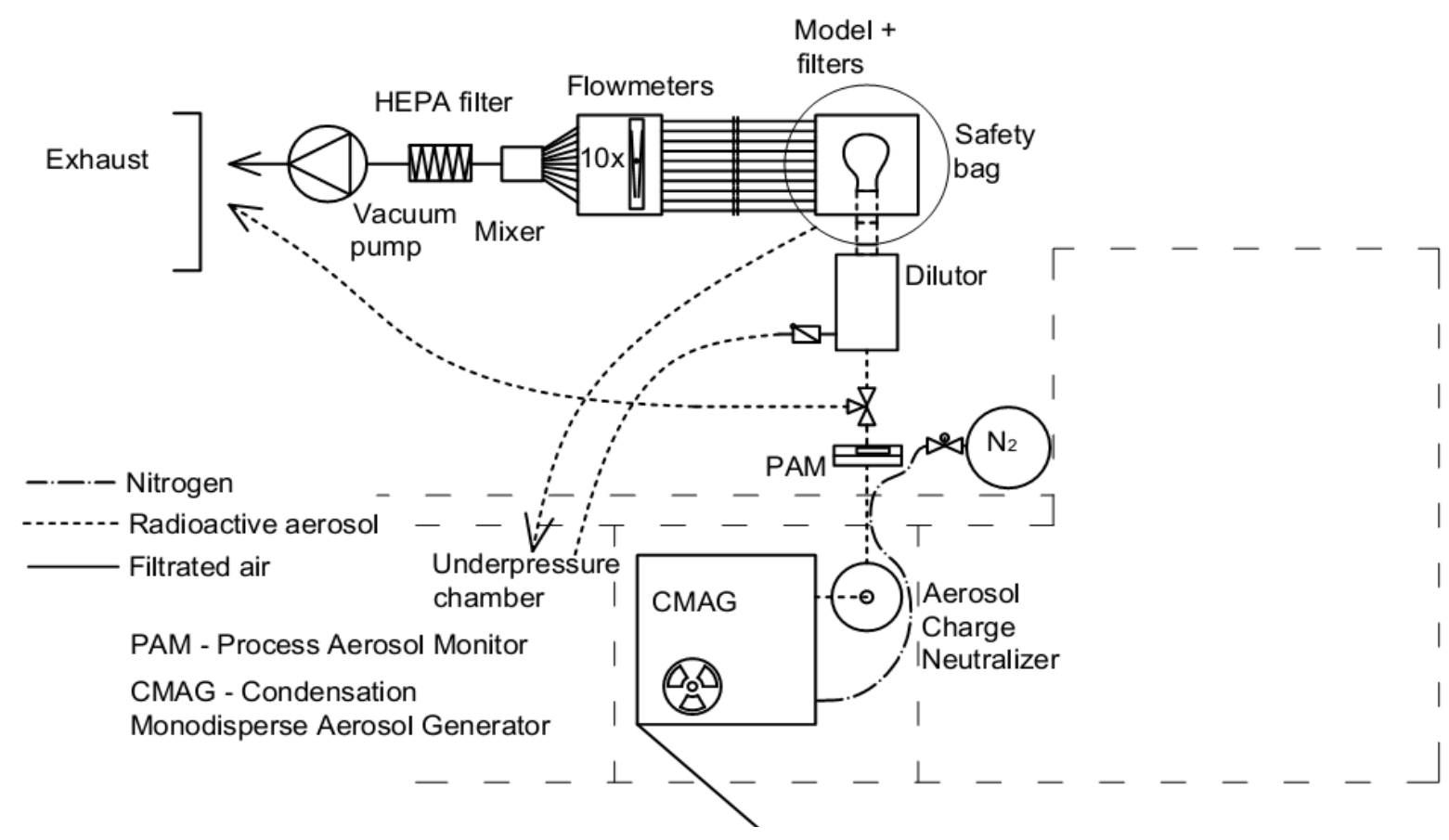

Fig. 2. A scheme of the setup for radioactive aerosol deposition measurement

\subsection{Numerical simulation}

The identical geometry of the realistic human airways was used also for the numerical simulations. The air flow was calculated using steady Reynolds-averaged NavierStokes (RANS) method with shear stress transport (SST) Menter's k- $\omega$ model of turbulence with treated boundary layer, which satisfies the conditions for low Reynolds number.

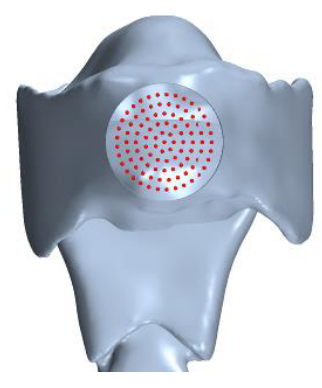

Fig. 3. The injection points of parcels representing particles in the numerical simulation.

The simulations were performed in a commercial solver Star-CCM+. The model had 6 million cells in polyhedral mesh. Particle deposition was simulated using embedded Lagrangian multiphase model with 10,000 parcels with particles of sizes $2.5,4.3,6.0$, and $8.0 \mu \mathrm{m}$. The particles were distributed to the model from a plane at the beginning of the inlet cylinder. The plane contains 100 equally distributed injection points (see Fig. 3). 100 parcels were emitted from each of the points. Initial velocity magnitude of the parcel is identical with the mean cross-sectional inlet velocity and direction is determined by a vector parallel with the axis of the inlet cylinder.

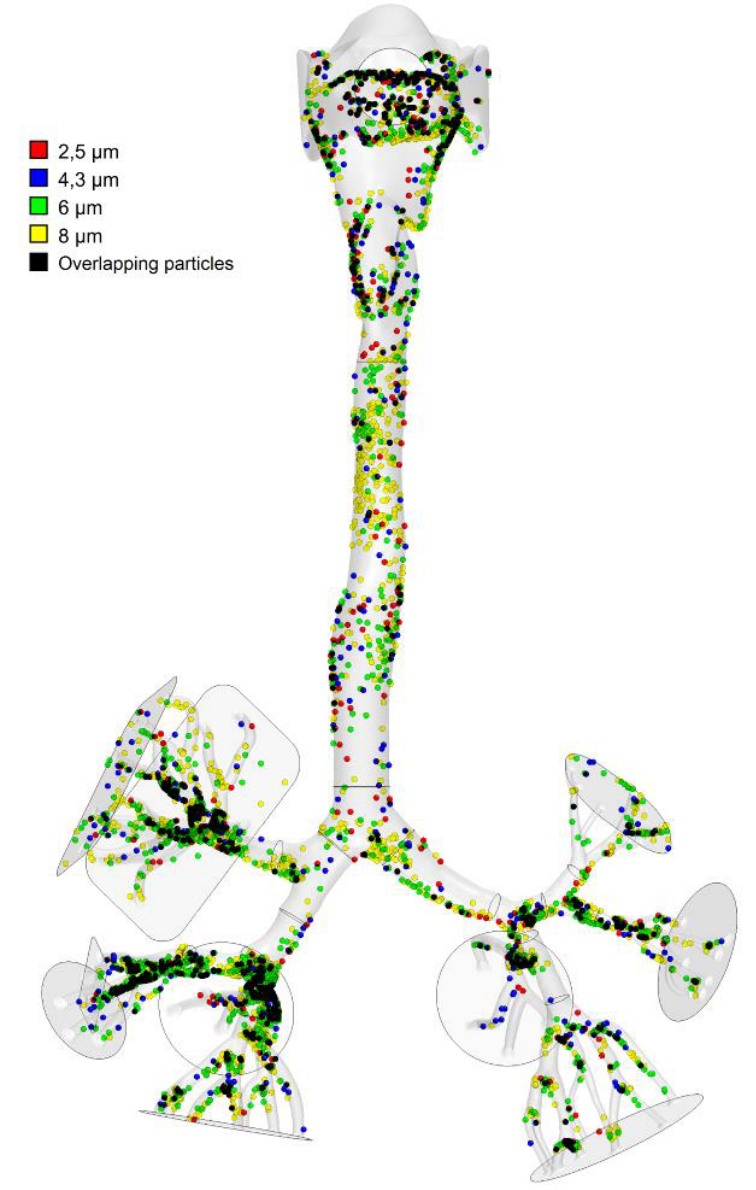

Fig. 4. The numerically simulated deposition sites of various sizes of particles for steady inhalation flow rate 30 liter/min. 


\section{Results and discussion}

As the numerical simulation enables more detailed tracing of particles compared to the experimental method, we plotted a figure with deposition sites of particles (see Fig. 4). In this way the possible deposition hot-spots could be identified. Particles of different sizes are represented by dots of a different colours. The deposition sites with overlaps of two or more different sizes of particles are represented by the black colour. It is obvious that the oral cavity presents a significant obstacle for the inhaled particles of all sizes. Then many particles also deposited in the glottal area. Interestingly, particles were separated based on their size downstream of the glottis. The biggest $8 \mu \mathrm{m}$ particles deposited in the upper part of the trachea, whereas the smaller $6 \mu \mathrm{m}$ particles deposited in the middle part. It is a consequence of the laryngeal jet, which arises in the glottis. As the biggest particles have the highest inertia, they cannot avoid the collision with the wall after being accelerated by the jet.

Surprisingly, only minor deposition occurred at the carina of the first bifurcation, where major deposition hotspot was expected. The particles apparently managed to follow the streamlines closely at this location for the flow rate of 30 liter/min and continued to the lower airways.

On the other hand, unexpectedly high local deposition was found in the entrance regions of segments 18,20 and 19. As all sizes of particles deposited there, it seems that not only the impaction but also turbulent dispersion influences the transport of particles.

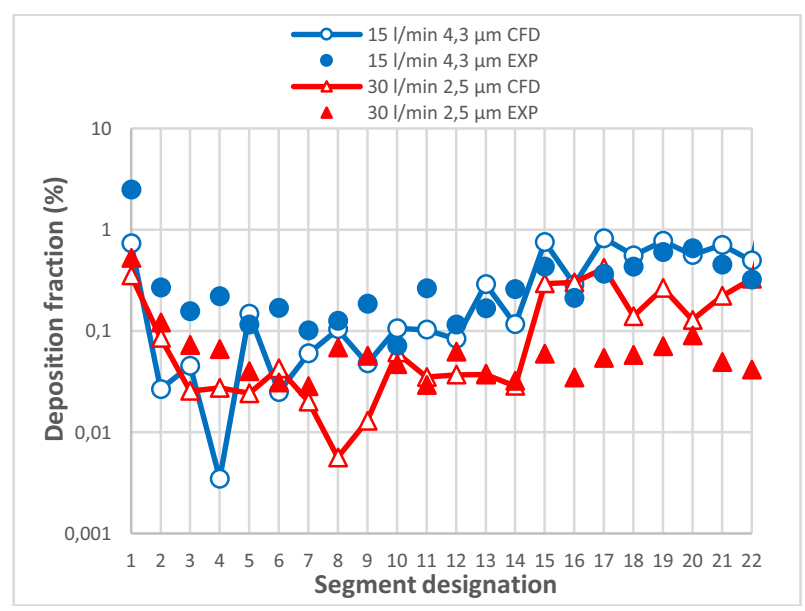

Fig. 5. A comparison of experimentally measured and numerically simulated deposition fractions in all segments of the realistic model of lungs.

However, the numerically simulated data does not agree well with the experimentally obtained data. As can be seen in Fig. 5, the simulation underestimates the deposition fraction in the upper part of the model (segments 1 to 11 ) for the lower flowrate (15 liter/min), whereas the segments with multiple branching (segments 12 to 22) were simulated correctly. On the contrary, for the higher flowrate (30 liter/min) the upper part (except the segment no. 8) was simulated with sufficient precision, but the lower segments were significantly overestimated by the simulation.
The above mentioned differences do not necessarily mean that the simulation is inaccurate. It should be noted that the Langrangian deposition model contains a stochastic element and as this simulation was performed only once, it must be seen as one of statistically random results. It is necessary to perform the simulation repeatedly and only after then calculate the average deposition fraction and compare the simulations with the experiments. This procedure will be therefore accomplished as a next step.

\section{Conclusions}

The comparison of experimental and numerically simulated deposition results demonstrated that the computational model was able to simulate general trends, although notable differences were found after the first run of simulation. Therefore, further repeated simulations are needed to receive statistically relevant data. Only after the successful validation can the numerical simulation serve for the prediction of localized aerosol deposition in human lungs.

Acknowledgement: This work was supported by the Czech Science Foundation under the grant GA16-23675S and by the project FSI-S-14-2355.

\section{References}

A. Churg and S. Vedal, Occup Environ Med 53 (8), 553 (1996).

W. Hofmann, Journal of Aerosol Science 42 (10), 693 (2011).

A. Farkas, A. Jokay, P. Furi, I. Balashazy, V. Muller, B. Odler, and A. Horvath, Aerosol and Air Quality Research 15 (6), 2466 (2015).

A. A. Rostami, Inhalation Toxicology 21 (4), 262 (2009).

A. Kerekes, A. Nagy, M. Veres, I. Rigo, A. Farkas, and A. Czitrovszky, Measurement 90, 144 (2016).

K. Bauer and C. Brucker, Journal of Biomechanical Engineering-Transactions of the Asme 137 (8) (2015). J. Elcner, M. Forman, J. Jedelský, F. Lízal, and M. Jícha, presented at the International conference Experimental Fluid Mechanics 2010, Liberec, 2010 (unpublished).

L. T. Holbrook and P. W. Longest, Journal of Aerosol Science 59, 6 (2013).

F. Lizal, M. Belka, J. Adam, J. Jedelsky, and M. Jicha, Proceedings of the Institution of Mechanical Engineers Part H-Journal of Engineering in Medicine 229 (10), 750 (2015).

10 F. Lizal, J. Elcner, P. K. Hopke, J. Jedelsky, and M. Jicha, Proceedings of the Institution of Mechanical Engineers Part H-Journal of Engineering in Medicine 226 (H3), 197 (2012).

11 A. Schmidt, S. Zidowitz, A. Kriete, T. Denhard, S. Krass, and H. O. Peitgen, Computerized Medical Imaging and Graphics 28 (4), 203 (2004).

12 Y. Zhou and Y. S. Cheng, Aerosol Science and Technology 39 (6), 492 (2005). 